\title{
Daten gewinnen, Wissen nutzen - eine gesundheitswissenschaftliche Herausforderung auch nach 250 Jahren
}

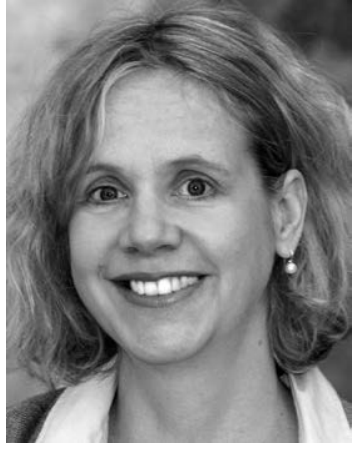

Prof. Dr. med. Julika Loss

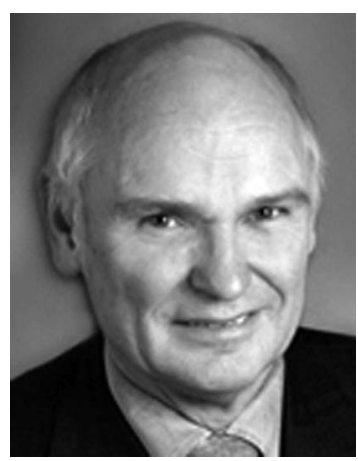

Dr. med. Gert von Mittelstaedt

Bibliografie

DOI http://dx.doi.org/ 10.1055/s-0035-1559784 Gesundheitswesen 2015; 77: 529-530

(c) Georg Thieme Verlag KG Stuttgart · New York

ISSN 0941-3790

Korrespondenzadresse

Prof. Dr. med. Julika Loss Medizinische Soziologie Institut für Epidemiologie und Präventivmedizin Fakultät für Medizin Universität Regensburg Dr. Gessler-Straße 17 93051 Regensburg Julika.Loss@klinik.uniregensburg.de
Werten wir das Experiment von James Lind zur Behandlung des Skorbut als Startschuss für systematische Studien, dann blicken wir heute auf mehr als 250 Jahre zurück, in denen sich die Datengewinnung für die medizinische Versorgung entwickelt hat. Der schottische Schiffsarzt Lind hatte 1747 zwölf an Skorbut erkrankte Matrosen in 6 Gruppen eingeteilt, denen jeweils eine unterschiedliche Behandlung zugeteilt wurde (z.B. Apfelwein oder Schwefelsäure). Lediglich 2 Seeleute, die Zitrusfrüchte erhielten, erholten sich wieder [1]. Es hat in der Folge lange gedauert, bis sich systematische Untersuchungen in größeren Patientengruppen entwickelten. Mit Einführung der Sozialgesetzgebung Ende des 19. Jahrhunderts wurden sozialmedizinische Daten unverzichtbar für die Funktion und die Weiterentwicklung unseres solidarisch organisierten Systems der gesundheitlichen Sicherung. Fortschritte im Fachgebiet Statistik, die Flut an neuen Medikamenten, die praktisch unkontrolliert auf den Markt drängten, und nicht zuletzt das Bekanntwerden von massiven Nebenwirkungen, z.B. im Rahmen der Contergan-Tragödie, führten dazu, dass in den 1960er Jahren der Goldstandard der randomisierten kontrollierten Studie für klinische Fragestellungen etabliert wurde [2]. Zeitgleich hat die Sozialmedizin frühzeitig Grundsätze des Datenschutzes im Bereich ihrer Begutachtungen eingeführt. In die 1990er Jahre fallen dann die Ankündigung des neuen Paradigmas der evidenzbasierten Medizin und die zunehmende Akzeptanz der gesundheitsbezogenen Lebensqualität als relevantes „Outcome“.

Diese Entwicklungen sind, wenn auch mit einiger Verzögerung, in der Präventions- und Versorgungsforschung angekommen. Interventionen der Prävention und Gesundheitsförderung werden zunehmend wissenschaftlich evaluiert; in den letzten Jahren wurden für den deutschen Raum mehrere Aufarbeitungen vorgelegt, die beschreiben, wie Daten für Qualitätsmanagement und Erfolgskontrolle gesundheitsförderlicher Maßnahmen gewonnen werden können $[3,4]$. Für das in Deutschland vergleichsweise junge Gebiet der Versorgungsforschung wurde 2009/10 ein 2-teiliges Memorandum in dieser Zeitschrift veröffentlicht, das methodische Grundprinzipien und Mindeststandards definiert $[5,6]$ und damit Anleitung für qualitative hochwertige Datengewinnung in der Versorgungsforschung gibt. Viele Beiträge, die für den diesjährigen gemeinsamen Kongress „Daten gewinnen, Wissen nutzen für die Praxis von Prävention und Versorgung“ (Regensburg) als Vorträge oder Poster angenommen wurden, legen von dieser Entwicklung Zeugnis ab.

All diese Fortschritte können die klinische Versorgung, die sozialmedizinische Begutachtung und die Praxis der Prävention wissenschaftlich fundierter machen und damit berechenbarer, sicherer, erfolgreicher und effizienter. Allerdings wird parallel zur Zunahme von Studien auch einen Zuwachs an „research waste“ beklagt [7]: eine erhebliche Vergeudung von Ressourcen bei der Gewinnung und Publikation von Daten entstehe z.B. durch irrelevante Forschungsfragen, unangemessene Methoden sowie durch Ergebnisse, die nicht oder nur verzerrt berichtet werden. Auch die Gewinnung von hochwertiger Evidenz wird von kritischen Stimmen begleitet. Ein wesentlicher Aspekt dabei ist die unüberschaubare Flut an Evidenz: die Zahl an wissenschaftlichen Publikationen und Leitlinien ist so groß, dass es für den Praktiker immer schwieriger wird, die relevanten Informationen herauszufiltern. Der Erfolg der systematischen Datenerhebung wird damit zu ihrem eigenen Misserfolg, wenn es um die Umsetzung des Wissens in die Praxis geht. Nicht zuletzt deswegen diskutieren Greenhalgh et al. in einem vielbeachteten Essay im British Medical Journal die evidenzbasierte Medizin gar als „movement in crisis“ [8].

Datengewinnung ist keine l'art pour l'art. Das generierte Wissen hat nur dann einen Sinn, wenn es Eingang in die Präventions- und Versorgungs-Praxis bzw. in politische Entscheidungen findet. Dieser Wissenstransfer ist eine mühsame und langwierige Angelegenheit - damals wie heute: die Seeleute mussten über 50 Jahre warten, bis nach Linds Experiment damit begonnen wurde, bei längeren Fahrten tägliche Zitronensaft-Rationen auszuteilen. Dieser „evidence-practice gap“ ist heute dank moderner Kommunikationsmethoden kürzer, aber dennoch oftmals noch erheblich, und betrifft neben der Medizin auch die Gesundheitswissenschaften. Für politische Entscheidungen im Bereich Public Health bzw. Prävention bspw. ist die akademische Literatur nur eine von vielen Informationsquellen neben z.B. Meinungen von Experten und Beratern [9]. Die Datengewinnung für Prävention und Versorgung muss daher die Komplexitäten von politischen Entscheidungen und Machbarkeiten in der Praxis berücksichtigen. Dazu gehören das soziale Gefüge, die Rechtslage und die Systemlogiken einzelner Settings und Regionen genauso wie Umsetzbarkeit und Akzeptanz in deren Bereich. Idealerweise werden Forschungsfragen und Lösungen von wissen- 
schaftlichen und gesellschaftlich-politischen Akteuren gemeinsam produziert - im Sinne einer so genannten „transdisziplinären Forschung“. Nowotny et al., die dieses Konzept in ihrem Buch „Re-thinking Science“ darlegen [10], stellen die Frage: Welchen Platz haben eigentlich die Menschen in den Erkenntnissen, die von der Wissenschaft produziert werden? Die medizinische Wissenschaft muss von den Bedürfnissen der Menschen ausgehen: in der Prävention von den Gesunden, in der Versorgung von den Kranken. Entsprechend ist eine angemessene Leistungsgewährung Gegenstand der sozialmedizinischen Begutachtung. Partizipative und qualitative Datengewinnung oder patienten-relevante Outcomes nehmen bewusst die Perspektive derjenigen ein, die Empfänger von Gesundheits- und Präventionsleistungen sind oder werden sollen, und erzeugen damit Wissen, das für den Alltag nutzbar gemacht werden kann. Die Abstracts in diesem Band liefern dafür viele positive Beispiele.

\section{Literatur}

1 Bhatt A. Evolution of Clinical Research: A History Before and Beyond James Lind. Perspect Clin Res 2010; 1: 6-10

2 Meldrum ML. A brief history of the randomized controlled trial. Hemat Oncol Clin North Am 2000; 14: 745-760

3 Tempel N, Reker N, Bödeker M et al. Qualitätssicherung in der Gesundheitsförderung in Settings. Ansätze, Charakteristika und Empfehlungen. Präv Gesundheitsförd 2013; 8: 73-77

4 Loss J, Eichhorn C, Reisig V et al. Qualitätsmanagement in der Gesundheitsförderung - Entwicklung eines multidimensionalen Qualitätssicherungsinstruments für eine landesweite Gesundheitsinitiative. Präv Gesundheitsförd 2007; 2: 199-206

5 Pfaff H, Glaeske G, Neugebauer EAM et al. Memorandum III: Methoden für die Versorgungsforschung, Teil 1. Gesundheitswesen 2009; 71: 505-510

6 Neugebauer EAM, Icks A, Schrappe M. Memorandum III: Methoden für die Versorgungsforschung, Teil 2. Gesundheitswesen 2010; 72: 739-748

7 Chalmers I, Glasziou P. Avoidable waste in the production and reporting of research evidence. Lancet 2009; 374: 86

8 Greenhalgh T, Howick J, Maskrey $N$. Evidence based medicine: a movement in crisis? BMJ 2014; 13: g3725

9 Orton L, Lloyd-Williams F, Taylor-Robinson D et al. The use of research evidence in public health decision making processes: systematic review. PLoS One 2011; 6: e21704

10 Nowotny H, Scott P, Gibbons M. Re-Thinking Science. Knowledge and the Public in an Age of Uncertainty. Oxford: Polity Press; 2001 